\title{
El sistema óptico de enfoque de Feynman
}

\author{
Feynman's optical focusing system
}

\author{
José Ignacio Castro ${ }^{10}$, Arturo López Dávalos ${ }^{* 2}$ \\ ${ }^{1}$ Universidad Nacional de San Juan, San Juan, Argentina \\ ${ }^{2}$ Universidad Nacional de Río Negro, Bariloche, Argentina
}

Recibida en 15 de Agosto, 2018. Revisado en 1 de Diciembre, 2018. Aceptado en 1 de Diciembre, 2018

\begin{abstract}
Analizamos el comportamiento de un sistema óptico de enfoque, utilizado por Richard Feynman en sus famosas clases de física. El sistema es una lente óptica biconvexa con simetría de revolución en torno a un eje, definida por la propiedad de que los rayos que parten de una fuente puntual, ubicada en un foco sobre el eje, convergen a un punto imagen al otro lado de la lente, de modo que las ondas luminosas sobre ellos llegan simultáneamente a este último punto. Esto asegura que las ondas de una frecuencia dada viajando según diferentes caminos desde un foco al otro a través de aire y vidrio, completan el mismo número de ciclos cuando alcanzan el punto imagen y por lo tanto interfieren en fase entre sí. En este trabajo mostramos explícitamente que el perfil de la lente de Feynman debe ser hiperbólico, forma que para rayos paraxiales elimina la aberración esférica (pero no la aberración cromática) de la lente.
\end{abstract}

Palabras clave: Óptica geométrica, Lente hiperboloidal, Principio de Fermat, Aberraciones

We analyze the behavior of an optical focusing system, used by Richard Feynman in his famous physics classes. The system is a biconvex optical lens with symmetry of revolution around an axis, defined by the property that the rays that start from a point source, located in a focus on the axis, converge to an image point on the other side of the lens, so that the light waves on them arrive simultaneously at this last point. This ensures that the waves of a given frequency, traveling according to different paths from one focus to the other through air and glass, complete the same number of cycles when they reach the image point and therefore interfere in phase with each other. In this work we explicitly show that the profile of the Feynman lens must be hyperbolic, a form that for paraxial rays eliminates the spherical aberration (but not the chromatic aberration) of the lens.

Keywords: Geometric optics, Hyperboloidal lens, Fermat's principle, Aberrations.

\section{Introducción}

En su libro Física, Richard Feynman [1] utiliza lo que llama un sistema óptico de enfoque, que en la práctica no es más que una lente óptica. Tal sistema tiene la propiedad de que los rayos que parten de una fuente puntual $S$ y convergen a un punto imagen $F$ del otro lado del vidrio arriban simultáneamente a $F$. Feynman usa un argumento similar en su libro Electrodinámica cuántica La extraña teoría de la luz y la materia [2] cuando hace referencia a la propagación de la luz y de los electrones.

Tal comportamiento asegura que las ondas de una frecuencia dada que viajan a lo largo dediferentes caminos que unen a la fuente $S$, ubicada en un foco, con el punto Fubicado en el otrofoco a través del aire y del cristal, completen el mismo número de ciclos cuando alcanzan el puntoimagen y por lo tanto están en fase entre sí, e interfieren constructivamente.

La propiedad invocada por Feynman se relaciona con el principio de Fermat, y es un problema cuyo estudio se remonta en el tiempo hasta el análisis de la refracción de la luz realizados por René Descartes a principios del siglo

*Endereço de correspondência: arturolopezdavalos@gmail.com.
XVII $[3 ; 4]$. Tal propiedad exige que las ondas empleen un tiempo extremo (mínimo) sobre el camino efectivo que toman para ir de un punto a otro; toda onda que satisface este requerimiento interfiere constructivamente con otras de la misma fase optimizando la propagación. En un sistema de enfoque, que produce una imagen real y puntual de un objeto puntual, cuando hay varios caminos posibles que satisfacen el criterio de Fermat la luz recorre todos ellos, y lo hace en el mismo tiempo mínimo. Tal es el caso del espejo elíptico, geometría para la cual sabemos que la suma de las distancias de un punto del espejo a los focos es siempre la misma, lo que le da a la luz que parte de un foco la posibilidad de recorrer múltiples caminos, tardando el mismo tiempo mínimo sobre todos ellos e interfiriendo constructivamente para formar imagen en el otro foco; el tiempo es mínimo respecto de trayectorias curvilíneas o poligonales diferentes de las mencionadas. Todos los rayos que salen de un foco en cualquier dirección convergerán al otro, a menos que limitemos los caminos posibles con colimadores o pantallas, en cuyo caso sólo los caminos libres serán efectivos para formar una imagen (con menor intensidad luminosa). 
Cabe señalar que la propiedad del sistema de enfoque de Feynman no es evidente para los alumnos cuando se trabaja en óptica de lentes, porque no se señala que el principio de Fermat implica la focalización con igualdad de fase; tal es el caso al tratar la difracción en las lentes, consideradas como elementos de estructura simple respecto de la fase [5].

Las lentes y los espejos reales son tradicionalmente construidos de forma esférica debido a la relativa facilidad mecánica para lograr esa forma. En particular, el pulido manual de los espejos de un telescopio produce superficies esféricas. Una desventaja de la forma esférica es el fenómeno de aberración esférica: un haz de rayos paraxiales (paralelos al eje óptico y cercanos al mismo) refractados, sólo pueden formar imagen en el foco de la lente si cortan al eje óptico en puntos distanciados a lo sumo media longitud de onda; las ondas sobre ellos tardan prácticamente el mismo tiempo (mínimo) para todo el haz e interfieren constructivamente en la imagen; otros rayos paralelos al eje óptico y refractados por fuera del haz paraxial dan lugar a la llamada aberración esférica pues forman imagen en puntos distanciados más de media longitud de onda del foco de la lente.

Por esto, es claro que una lente esférica sencilla no puede hacer el trabajo requerido por Feynman. A pesar de la popularidad del argumento de Feynman, y del tiempo transcurrido desde la publicación de su libro, no sabemos de ningún cálculo, en la literatura moderna, que indique explícitamente la forma que esa lente ideal debe tener. Como pensamos que es un tema interesante para tratar en clase, en este breve trabajo mostramos que la propiedad básica enunciada por Feynman determina la forma de la lente y que ésta corresponde a una lente hiperboloide de revolución.

Estas lentes son conocidas en relación con diversos sistemas ópticos por que evitan aberraciones como coma y astigmatismo que se presentan en los sistemas esféricos [6], pero debe señalarse que aún con lentes hiperbólicas el problema de las aberraciones aparece para puntos objeto alejadas del eje óptico, con ángulos de incidencia tan grandes que no pueden considerarse paraxiales. El modo de evitarlas exige, comúnmente, arreglos de lentes combinadas de modo tal que unas y otras compensan sus efectos indeseados.

En la literatura científica francesa la ley de refracción de Snell se conoce como ley de Snell-Descartes, como homenaje a la contribución de René Descartes al enunciado de la ley. En un libro de publicación relativamente reciente [7], el autor revisa la correspondencia de Descartes desde Holanda con un artesano de París, en la que lo instruye sobre cómo construir una máquina rectificadora para tallar una lente hiperbólica, lo cual muestra que Descartes ya había encontrado la solución a este problema, analizando el proceso de refracción.

\section{El Principio de Fermat y la Lente Hiperboloidal}

Por simplicidad, nos proponemos analizar una lente con simetría bilateral respecto del plano principal (yz) (en coordenadas cartesianas) y simetría de revolución respecto del eje óptico $x$, como se muestra en la Figura 1. Con referencia a dicha Figura 1 vemos que el tiempo necesario para recorrer el camino $S P_{S} P_{F} F$ en el plano (xy) está dado por

$$
T(|x|, y)=\frac{2}{c}\left[\sqrt{(d-|x|)^{2}+y^{2}}\right]+\frac{2 n}{c}|x|
$$

donde $c$ es la velocidad de la luz en el aire y $n$ es el índice de refracción relativo del vidrio. El uso de $|x|$ en esta expresión corresponde al hecho que $P_{S}$ es el primer contacto de la luz con la interfase aire-vidrio, cercana a la fuente; si no hiciéramos explícito el signo de la posición de dicho punto, no se le podría distinguir de $P_{F}$ (segundo contacto, con la interfase cercana al foco) y los caminos $S P_{S} P_{F} F$ y $S P_{F} P_{S} F$ resultarían confundidos. Para $|x|=e, y=0$, la ecuación (1) nos da el tiempo que tarda la luz ir entre $S$ y $F$ a lo largo del eje de simetría, eje óptico, del sistema es:

$$
T(e, 0)=\frac{2}{c}[d+(n-1) e]=\frac{2}{c} \alpha
$$

Para determinar el perfil de la lente que satisfaga el requerimiento de Feynman (todos los rayos tardan el mismo tiempo para ir de $S$ a $F$, imponemos la condición

$$
T(|x|, y)=T(e, 0)
$$

Igualando ahora (1) y (2) obtenemos la ecuación implícita que define el perfil de la lente:

$$
f(|x|, y)=\sqrt{(d-|x|)^{2}+y^{2}}+n|x|-\alpha=0
$$

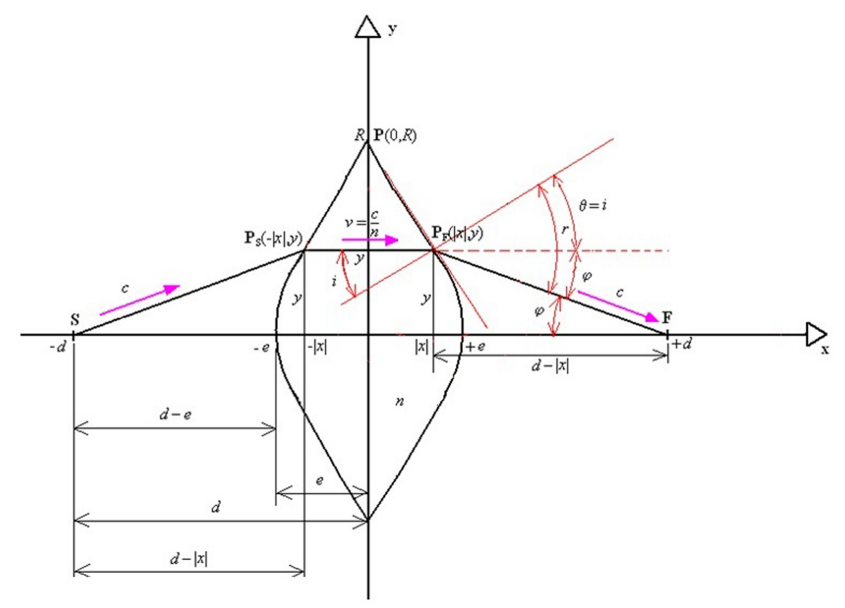

Figura 1: Lente hiperbólica y las variables usadas en el texto. 
Reordenando términos obtenemos la igualdad:

$$
\sqrt{(d-|x|)^{2}+y^{2}}=\alpha-n|x|
$$

y desarrollando:

$(d-|x|)^{2}+y^{2}=(\alpha-n|x|)^{2}=\alpha^{2}-2 \alpha n|x|+n^{2}|x|^{2}$

Completando cuadrados esta expresión puede llevarse a una forma más conocida:

$$
\frac{\left(|x|-x_{C}\right)^{2}}{a^{2}}-\frac{y^{2}}{b^{2}}=1
$$

es decir una hipérbola. Las coordenadas del centro de la hipérbola son (ver Figura 2)

$$
\begin{array}{r}
x_{c}=\frac{d+n e}{n+1} \\
y_{c}=0
\end{array}
$$

Los semiejes $a$ y $b$ están dados por $a=\frac{d-e}{n+1}$ y $b=$ $a \sqrt{n^{2}-1}=\frac{d-e}{n+1} \sqrt{n^{2}-1}$

Podemos identificar las dos caras de la lente con dos hipérbolas una centrada en $+x_{C}$ y otra centrada en $-x_{C}$, cuyos focos se indican son $F$ ty $F$ y $S$ y $S$ ten la Figura 2. Como se observa en la Figura 2, las caras derecha e izquierda de la lente quedan determinadas por hiperboloides de revolución cuyas intersecciones con el plano $(x y)$ vienen dadas, para cada una, por una rama de cada hipérbola, centradas en $\left( \pm x_{c}, 0\right)$ y definidas por las ecuaciones:

$$
\frac{\left(x \mp x_{c}\right)^{2}}{a^{2}}-\frac{y^{2}}{b^{2}}=1
$$

Los focos geométricos de las hipérbolas son $S, S, F y F$ y los focos ópticos son $S$ y $F$. Los vértices, de izquierda a derecha son $-x_{C}-a,-x_{C}+a x_{C}-a y x_{C}+a$.

Un resultado interesante es que la excentricidad $\epsilon$ de las hipérbolas está dada directamente por el índice de refracción del vidrio $\varepsilon=\sqrt{1+\frac{b^{2}}{a^{2}}}=n$.

Una lente hiperboloidal resulta de la revolución (en torno al eje óptico $x$ ) de las dos ramas hiperbólicas de trazo lleno en la Figura 2; el radio de abertura $R$ de la lente está determinado por los puntos $x=0, y=$

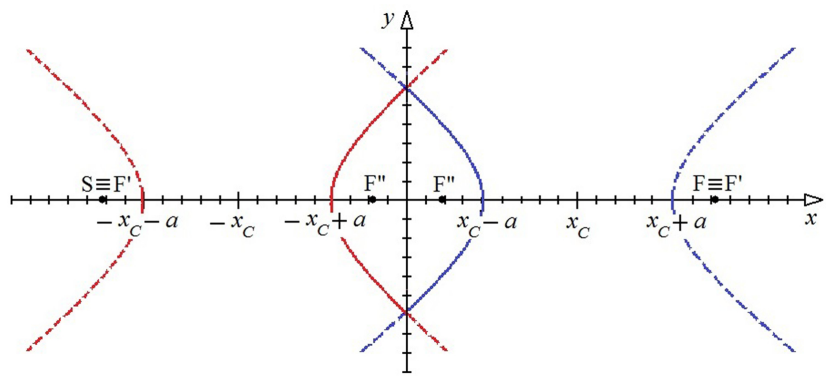

Figura 2: Las dos hipérbolas que determinan la lente, donde se han señalado los cuatro focos geométricos. $\pm R$ donde las hipérbolas correspondientes a las caras de la lente se intersectan y resulta

$$
R=\sqrt{\frac{n-1}{n+1}} \sqrt{(d+n e)^{2}-(d-e)^{2}}
$$

Este radio determina la luminosidad de la imagen, pues fija la cantidad de luz capturada por la lente. Vemos de estas relaciones que la distancia $2 d$ que separa los focos simétricos $S$ y $F$ de la lente hiperbólica está dada en términos del radio de abertura $R$, el semi-espesor $e$ de la lente y el índice de refracción $n$ por

$$
2 d=\frac{(n+1)\left[R^{2}-e^{2}(n-1)^{2}\right]}{e(n-1)^{2}}
$$

\section{Ley de Snell Para la Lente Hiperboloidal}

Notamos que en la deducción de la sección anterior no hemos hecho uso de la ley de Snell, sino de la condición de tiempos iguales para todos los rayos, invocada por Feynman. Pero es claro que esa ley debe cumplirse, lo que demostraremos a continuación. Para ello notamos que en el primer y cuarto cuadrantedel plano $(x, y)$, la variable $x$ es positiva, y por ello $|x|=x$ para el punto genérico $P(x y)$ de la cara derecha de la lente; como dicha rama satisface $(3)$ tenemos:

$$
f(x, y)=\sqrt{(d-x)^{2}+y^{2}}+n x-\alpha=0
$$

Calculamos la pendiente $m$ de este perfil en el punto $P_{F}$,

$$
\frac{d y}{d x}=m=-\tan \left(\frac{\pi}{2}-\theta\right)=-\frac{1}{\tan \theta}
$$

$(\operatorname{con} \theta>0)$. Es posible obtener la forma explícita de $y=y(x)$ mediante la fórmula para las raíces de la ecuación cuadrática, y de ella la derivada $d y / d x$, pero lo haremos evaluando dicha derivada a partir de la forma implícita (3), como ejercicio (poco frecuente) de la derivación de funciones implícitas, ejercicio que no implica mayor dificultad. Tenemos así:

$$
\frac{\partial f}{\partial x} d x+\frac{\partial f}{\partial y} d y=d \alpha=0 \rightarrow m=\frac{d y}{d x}=-\frac{\left(\frac{\partial f}{\partial x}\right)}{\left(\frac{\partial ? ?}{\partial y}\right)}
$$

Las derivadas parciales son:

$$
\frac{\partial f}{\partial x}=-\frac{(d-x)}{\sqrt{(d-x)^{2}+y^{2}}}+n
$$

y

$$
\frac{\partial f}{\partial y}=\frac{y}{\sqrt{(d-x)^{2}+y^{2}}}
$$


con lo cual resulta

$$
\begin{aligned}
m=\frac{d y}{d x} & =-\frac{\left(\frac{\partial f}{\partial x}\right)}{\left(\frac{\partial f}{\partial y}\right)}=\frac{-\frac{(d-x)}{\sqrt{(d-x)^{2}+y^{2}}}+n}{\frac{y}{\sqrt{(d-x)^{2}+y^{2}}}} \\
& =\frac{(d-x)}{y}-n \sqrt{\left(\frac{d-x}{y}\right)^{2}+1}
\end{aligned}
$$

La pendiente $m \prime=\tan \varphi$ del rayo $P_{F} F$, en la Figura 1 $(\operatorname{con} \varphi>0)$, es a su vez

$$
m \prime=-\frac{y}{(d-x)}
$$

y usando (8) y (9) encontramos la relación

$$
\begin{aligned}
m= & -\frac{1}{m \prime}-n \sqrt{\frac{1}{m^{\prime 2}}+1} \\
& \text { o bien } \\
& \frac{1}{\tan \theta}+\frac{1}{\tan \varphi}=n \sqrt{\frac{1}{\tan ^{2} \varphi}+1}
\end{aligned}
$$

que provee una relación entre $\theta$ y $\varphi$ mediada por el índice de refracción $n$. Luego de algunas trasformaciones simples arribamos a

$$
n=\frac{\sin (\theta+\varphi)}{\sin \theta}=\frac{\sin r}{\sin i}
$$

donde hemos llamado $r$ (ángulo de refracción) a $\theta+\varphi$ e $i$ (ángulo de incidencia) a $\theta$ como se ve en la Figura 1. Tal como podíamos esperar, esta es la ley de Snell en el punto $P_{F}$, donde debe recordarse que se pasa del vidrio al aire y por lo tanto el ángulo de incidencia es menor que el de refracción.

\section{Conclusión}

Hemos encontrado la forma explícita del perfil de la lente hiperboloidal que realiza las propiedades del sistema óptico de enfoque propuesto por Feynman, a partir del principio de Fermat, principio que es no local porque se refiere a la trayectoria completa de los rayos, y hemos verificado como dicha lente satisface a la ley de Snell, que es local porque se refiere a los puntos de la interfase airevidrio. Asimismo, hemos visto como la distancia entre los focos depende de la abertura, el espesor y el índice de refracción de la lente, mientras que la excentricidad de las hipérbolas de perfil depende sólo del índice de refracción del material de la lente.

Por otra parte es evidente que, por la simetría del sistema, si las ondas deben alcanzar el foco $F$ simultáneamente, todas ellas pasarán simultáneamente por el plano principal $x=0$; por tanto, debido a la reversibilidad del camino óptico, tardarán el mismo tiempo en llegar hasta allí, lo cual significa que la primera cara refractante (izquierda) produce la imagen de $S$ en $x \rightarrow+\infty$ porque todos los rayos pasan perpendicularmente al plano $x=0$, condición necesaria de simetría.

Si relajáramos la condición de simetría respecto al plano $x=0$ los rayos dentro del vidrio seguirán siendo paralelos al eje y la segunda cara refractante (derecha) puede ser diferente de la primera. Sin embargo debe ser del mismo tipo hiperbólico, pero con el foco $F$ en una nueva posición sobre el eje $x$; la rama izquierda de la hipérbola centrada en $+x_{C}$ determinará la cara derecha de la lente, como superficie de un hiperboloide de revolución con nuevos parámetros. La ecuación (7) muestra que el sistema no elimina la aberración cromática dado que la distancia entre focos depende del índice de refracción, que a su vez depende del color (es decir, de la longitud de onda involucrada).

Concluimos este trabajo agradeciendo a RBEF su amable recepción, así como las valiosas observaciones del revisor, corrigiendo, modificando y complementando el manuscrito original.

\section{Referencias}

[1] R.P. Feynman, R.B. Leighton y M. Sands, Física: Mecánica, radiación y calor (Addison-Wesley Iberoamericana, Wilmington, 1987), v. 1.

[2] R.P. Feynman, Electrodinámica cuántica - La extraña teoría de la luz y la materia (Alianza Editorial, Buenos Aires, 1989).

[3] E. Hecht y A. Zajac, Optica (Addison-Wesley Iberoamericana, Wilmington, 1986).

[4] E. Hecht, Optics (Pearson, Londres, 2017), $5^{\mathrm{a}}$ ed.

[5] J.W. Goodman, Introduction to Fourier Optics (McGraw Hill, New York, 1996), $2^{\mathrm{a}}$ ed.

[6] A. Camacho, Revista Mexicana de Física 45, 315 (1999).

[7] D.G. Burnett, Descartes and the hyperbolic quest: lens making and their significance in the seventeenth century (American Philosophical Society, New York, 2005). 